\title{
Distribución sociométrica en las aulas de chicos y chicas a lo largo de la escolaridad
}

\author{
FranCisCo-JuAn García-BACETE ${ }^{1}$, INMACUlada GarCía ${ }^{2}$ \\ E INÉS MONJAS ${ }^{3 *}$ \\ ${ }^{1}$ Universidad Jaume I; ${ }^{2}$ Universidad de les Illes Balears; ${ }^{3}$ Universidad de Valladolid
}

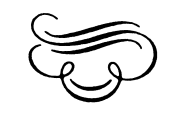

\section{Resumen}

La muestra está formada por 2173 alumnos escolarizados (45.7\% eran chicas y el 54.3\% chicos) en 92 aulas de todos los cursos desde el segundo ciclo de infantil al primer ciclo de secundaria, de 24 colegios públicos de Castellón, Valladolid y Palma de Mallorca.

Chicos y chicas son sociométricamente diferentes en todos los ciclos. Ellos son más rechazados y controvertidos y ellas más ignoradas y promedio. Sólo la proporción entre chicos-controvertidos y chicas-controvertidas varía en la escolaridad. La distribución sociométrica de los chicos es estable durante toda la escolaridad, la de las chica cambia (menos preferidas al principio y final de la escolaridad y más controvertidas en secundaria). El rechazo está asociado al género masculino. Hay variaciones en la distribución sociométrica de un aula a otra.

Palabras clave: Sociometría en el aula, género, curso.

\section{Sociometric distribution of boys and girls during all the schooling period}

\begin{abstract}
The sample is formed by 2173 students (45,7\% girls and 54,3\% boys) distributed in 92 classrooms, from the second cycle of preschool to the first cycle of secondary education, of 24 public schools in Castellon, Valladolid and Palma de Mallorca. Boys and girls are sociometrically different in all the cycles. In general terms, boys are more rejected and controversial than girls, and girls are more neglected and average than boys. Only the proportion between controversial-boys and controversial-girls changes in the schooling. The sociometric distribution for boys is stable during all the schooling period, while the distribution for girls changes from less popular in preschool and the last cycle of school to more controversial in secondary school. Rejection is related to males. However, there are great variations in the sociometric distribution from one classroom to another.

Keywords: Sociometry in the classroom, gender, school year
\end{abstract}

Agradecimientos: Investigación realizada gracias a las ayudas de investigación "El rechazo entre iguales en la vida
cotidiana" (SEJ2004-04028) concedida por el Ministerio de Ciencia y Tecnología (Plan Nacional de
I+D+I,2004-07) y "El rechazo entre iguales: situación en los centros de infantil y primaria de la provincia de
Castellón" (P1-1B2003-28) concedida por Fundació Bancaixa-Universitat Jaume I.
Correspondencia con los autores: Francisco-Juan García Bacete. Departamento de Psicología Evolutiva, Educativa,
Social y Metodología. Facultad de Ciencias Humanas y Sociales. Universitat Jaume I. Avda Vicente Sos Baynat
s/n. 12071 Castellón. Teléfono: 964-729549. Fax: 964-729262. E-mail: fgarcia@ psi.uji.es
*Miembros del Grupo GREI (Grupo de Investigación del Rechazo Entre Iguales en el Contexto Escolar) 
Los iguales proporcionan a los niños y niñas oportunidades para practicar tanto la independencia respecto al control de los adultos como relaciones más simétricas con otros, compartir valores e intercambiar apoyo emocional (Chan y Mpofu, 2002). En el contexto escolar, los iguales ofrecen a los niños tanto una sensación de pertenencia como diversas formas de intercambio académico (intercambiar apuntes, realizar juntos los deberes escolares,...) (Wentzel y Asher, 1995). El valor que se otorga a las normas de grupo y los diversos mecanismos que los grupos ejercen sobre los individuos para que se conformen con ellas añade, si cabe, mayor importancia a las relaciones sociales en la infancia y sobretodo durante la adolescencia (Oliva, 1999). Las relaciones entre iguales en general, y el estatus sociométrico en particular, tienen un impacto único en el desarrollo de una identidad y autoestima personal, en las relaciones sociales presentes y futuras, en la vida académica de los alumnos y en su competencia escolar. La capacidad de los compañeros para predecir el ajuste personal, social y académico futuro de un alumno ha despertado un gran interés por la perspectiva sociométrica (Cillessen y Bukowski, 2000). Su técnica fundamental es el test sociométrico de nominaciones directas (Arruga, 1983; Rodríguez y Morera, 2001).

Los procedimientos de clasificación sociométrica se diferencian en estandarizados y de probabilidad, siendo éstos últimos los que ofrecen más ventajas (García-Bacete, 2006, 2007). La tipología sociométrica más extendida es la que identifica a los alumnos como preferidos, rechazados, ignorados, controvertidos y adaptados o promedios, de acuerdo con unos criterios en relación al número de nominaciones positivas y negativas recibidas.

El mayor volumen de investigaciones ha intentado averiguar las características de los tipos sociométricos mediante el estudio de sus correlatos conductuales y emocionales (Cava y Musitu, 2001; Ortíz, Aguirrezabala, Apodaka, Etxebarria y López, 2002; Jiménez, 2003; Trianes, Blanca, Muñoz, García, Cardelle-Elawae e Infante, 2002), aunque cada vez más se incrementan los trabajos que incorporan otras variables y modelos (García-Bacete, Musitu y García, 1990; Villanueva, Clemente y García-Bacete, 2001). Los resultados del metaanálisis realizado por Newcomb, Bukowski y Pattee (1993) muestran claramente que los niños de cada tipo sociométrico tienen un repertorio conductual que influye en la calidad de sus relaciones interpersonales. García-Bacete (2007) ofrece la siguiente caracterización de cada tipo. Los alumnos rechazados son claramente los más agresivos y aislados y los menos sociables. Por su parte, los alumnos preferidos son claramente los más sociables, los menos aislados y sus tasas de agresividad son tan bajas como las de los ignorados. Los alumnos ignorados tienen niveles muy bajos de agresividad, bajos de sociabilidad y entre bajo y medio-bajo en aislamiento. Los alumnos controvertidos, como los rechazados, siempre tienen tasas por encima de la media en agresividad y aislamiento, pero son menos sociables que los preferidos en la muestra de pequeños.

Los porcentajes para un mismo tipo varían de unos estudios a otros. A modo orientativo, hemos establecido los siguientes intervalos (García-Bacete, 2006b): entre $10-15 \%$ para preferidos y rechazados, ligeramente superiores los ignorados $(12-17 \%)$ y porcentajes menores de controvertidos (2-6\%). Los porcentajes y características de los diferentes tipos varían en función del género de los alumnos, y en menor medida de la edad. Cillesen, Coie, Terry y Lochman, (1996) señalan que los niños tienen el doble de probabilidad de ser rechazados que las niñas y la mitad de ser preferidos. Las niñas tienen más probabilidades de ser ignoradas y menos de ser identificadas como controvertidas que los niños.

Duncan y Cohen (1995) afirman que los niños eligen a sus amigos buscando similitudes con sus propias características, por ejemplo, edad, género, logro académico, agresividad o tipo sociométrico. En este sentido no es de extrañar que 
los subgrupos estén formados por niños de edades parecidas, o que preferidos y no-preferidos formen sus propios subsistemas o que se elija preferentemente a los del mismo género. En opinión de Legault (1993), el fenómeno de "segregación de género" en las aulas, sobretodo en primaria, es una realidad muy extendida. García-Bacete (2006) confirma tanto el carácter intragénero de las nominaciones positivas como el sesgo hacia los chicos de las nominaciones negativas.

Cillesen et al. (1996) afirman que a las niñas sus compañeros y compañeras les suelen atribuir más conductas prosociales y de aislamiento que a los niños, y a éstos más de agresión y de "meterse en líos" que a las niñas. No obstante, se observan diferencias a nivel de prioridad en los motivos que unos y otros dan para elegir a quienes les gusta más o menos como amigos, o en los motivos por los que unos y otras son aceptados o excluidos (García-Bacete, García, Sureda, 2005; García-Bacete, Lara y Monjas, 2005). Por ejemplo, los chicos rechazan más frecuentemente que las chicas a alguien que molesta, pega o es mal estudiante, mientras que para las chicas la falta de reciprocidad, el mal carácter, el aburrimiento o la ausencia de relación son razones de más peso. En la misma línea, Duncan y Cohen (1995) señalaron que las valoraciones que se hacen de los compañeros dependen del tipo sociométrico y del género del evaluador.

Con la edad se producen una serie de cambios que pueden afectar las relaciones sociales y la tipología sociométrica. La percepción de la conducta de los iguales cambia con la edad. Así, por ejemplo, el comportamiento agresivo suele ser siempre una conducta de las que no gustan, pero las conductas de aislamiento como "jugar solo" sólo empiezan a percibirse como algo que no gusta a partir de los 8-10 años, de forma que los "aislados-pasivos" se convierten en "aislados-activos" (Hatzichriston y Hopf, 1996). Legault (1993) afirma que tanto niños como niñas se muestran cada vez más expansivos y precisos en la representación de las redes sociales en un aula. Los diferentes tipos sociométricos se hacen más visibles y diferenciados, en particular los rechazados (García-Bacete 2007). Así, los ignorados son cada vez un poco más sociables pero también más aislados y los controvertidos siguen siendo tan agresivos como los rechazados pero se vuelven significativamente más habilidosos que éstos.

\section{Objetivos}

El objetivo principal es conocer la distribución de los tipos sociométricos en las aulas a lo largo de la escolaridad. De forma más específica nos proponemos responder a las siguientes preguntas:

¿Cuántos niños y niñas de cada tipo sociométrico hay en cada período educativo? Hasta ahora las investigaciones han presentado los porcentajes de cada tipo sociométrico, bien referidos a grandes periodos (p.e., primaria, infancia,...), bien referidos a periodos concretos, sin relacionar los unos con los otros. Se trata por tanto de ofrecer conjuntamente, con una muestra amplia de aulas y alumnos, los porcentajes sociométricos distribuidos por género en cada uno de los ciclos educativos y en todo el periodo educativo a la vez. La hipótesis que mantenemos es que los porcentajes de cada tipo varían a lo largo de la escolaridad.

¿La distribución sociométrica es igual para los chicos que para las chicas? Nuestra hipótesis es que la proporción de chicos y chicas en cada tipo sociométrico es diferente. En concreto, esperamos encontrar más chicos rechazados y más chicas ignoradas.

¿La distribución sociométrica varía a lo largo de la escolaridad para niños y niñas? En concreto, esperamos encontrar efectos de interacción entre las variables ciclo x tipo sociométrico x género. Algunas de las hipótesis concretas son que la distribución de tipos a lo largo de la escolaridad varía en el caso de las chicas, que 
la proporción de controvertidos y controvertidas disminuye de infantil a secundaria, que en todos los ciclos la proporción de rechazados es superior al de rechazadas.

¿Todas las aulas de un mismo periodo educativo tienen la misma distribución sociométrica? Nuestra hipótesis es negativa. Esperamos encontrar distribuciones sociométricas diferentes en cada una de las aulas confirmando el impacto de las dinámicas sociales de cada aula. Al mismo tiempo, esperamos que las tendencias expresadas en los tres objetivos anteriores se mantengan en términos globales.

\section{Muestra}

La muestra está formada por 2173 alumnos escolarizados en 92 aulas de 24 colegios públicos de Castellón, Valladolid y Palma de Mallorca. El $45.7 \%$ eran chicas y el $54.3 \%$ chicos. La distribución por ciclos escolares es la siguiente: segundo ciclo de infantil (19 Aulas, 239 chicos, 199 chicas), ciclo inicial de primaria (33 Aulas, 374 chicos, 341 chicas), ciclo medio de primaria (14 Aulas, 181 chicos, 153 chicas), ciclo superior de primaria (21 Aulas, 295 chicos, 234 chicas), primer ciclo de secundaria ( 5 Aulas, 90 chicos, 67 chicas).

La participación de los centros y del profesorado ha sido voluntaria. La preocupación básica de los investigadores era disponer de una muestra amplia y diversa que permitiera cumplir con los objetivos propuestos. Esto es, poder disponer de un número elevado de aulas y que las aulas estuvieran distribuidas en el mayor número de colegios y contextos posibles. El que los investigadores residan en tres comunidades autónomas diferentes ha asegurado que los centros de la muestra posean características heterogéneas.

\section{Instrumento y procedimiento de identificación de los tipos sociométricos}

Cuestionario sociométrico. Incluye las siguientes preguntas: ¿Quiénes son los tres compañeros y compañeras de esta clase que eliges como mejores amigos o amigas? ¿Quiénes son los tres compañeros y compañeras de esta clase que menos te gustan como amigos o amigas?

El cuestionario de nominaciones entre iguales ha mostrado una adecuada fiabilidad test-restest, estructura factorial y validez convergente (González, 1998). Como sistema de clasificación hemos utilizado el procedimiento de probabilidad binomial continua propuesto por García-Bacete $(2006,2007)$, que mejora los procedimientos anteriores. El cálculo de los límites ha sido realizado con el programa Socio de González (1990). La clasificación se consigue aplicando los siguientes criterios:

- Preferidos: Np $\geq$ LS (Np) y Nn $<$ M (Nn)

- Rechazados: $\mathrm{Nn} \geq \mathrm{LS}(\mathrm{Nn})$ y $\mathrm{Np}<\mathrm{M}(\mathrm{Np})$

- Ignorados: $\mathrm{Np} \leq 1$ (en el caso de 5 elecciones el valor será 2) y $\mathrm{Nn}<\mathrm{M}(\mathrm{Nn})$

- Controvertidos: cumplen $[\mathrm{Np} \geq \mathrm{LS}(\mathrm{Np})$ y $\mathrm{Nn} \geq \mathrm{M}(\mathrm{Nn})]$ o $[\mathrm{Nn} \geq \mathrm{LS}(\mathrm{Nn})$

$\mathrm{y}-\mathrm{Np} \geq \mathrm{M}(\mathrm{Np})]$

- Medios: cumplen $[\mathrm{Np}<\mathrm{LS}(\mathrm{Np})$ y $\mathrm{Np}>1$ y $\mathrm{Nn}<\mathrm{LS}(\mathrm{Nn})]$ o $[\mathrm{Nn}<\mathrm{LS}$ (Nn) y $\mathrm{Nn} \geq \mathrm{M}(\mathrm{Nn})$ y $\mathrm{Np} \leq 1$ ] (en el caso de 5 elecciones el valor será 2 )

\section{Resultados}

\section{¿Cuántos niños y niñas de cada tipo sociométrico hay en cada ciclo de escolaridad?}

En la tabla I se presentan los porcentajes de cada tipo sociométrico para toda la muestra y desglosados por género y por ciclos. La mayor parte de alumnos son 
identificados como promedio (62.1\%). Casi un $10 \%$ son preferidos. El $28 \%$ restante es identificado negativamente por los compañeros: $4.2 \%$ son controvertidos, $11.3 \%$ son rechazados y $12.4 \%$ son ignorados. Como se observa en la tabla, los porcentajes son distintos para los chicos que para las chicas, así por ejemplo, el $32 \%$ de los chicos son identificados con tipos no-adaptativos frente al $23.1 \%$ de las chicas. También se observan variaciones a lo largo de la escolaridad dentro de cada género, especialmente en el caso de las chicas (por ejemplo, el porcentaje de preferidas oscila entre un $1.5 \%$ y un $12.4 \%$ ) y en los porcentajes relativos entre chicos y chicas (por ejemplo, mientras que en infantil el porcentaje de preferidos es similar entre chicos y chicas, $7.1 \%$ frente a $6.5 \%$, hay diferencias importantes en el ciclo superior, $7.8 \%$ frente al $1.5 \%$ ).

TABLA I

Porcentaje de cada tipos sociométrico por ciclo educativo y género

\begin{tabular}{|c|c|c|c|c|c|c|c|}
\hline & & \multicolumn{5}{|c|}{ CICLO EDUCATIVO } & \multirow[b]{2}{*}{ TOTAI } \\
\hline & & $2^{\circ}$ Infantil & Inicial & Medio & Superior & $1^{\circ} \mathrm{ESO}$ & \\
\hline \multirow[t]{5}{*}{ CHICOS } & Preferido & 7.1 & 9.4 & 11.6 & 11.6 & 7.8 & 9.7 \\
\hline & Rechazado & 14.6 & 16.8 & 16 & 16 & 15.6 & 16.0 \\
\hline & Ignorado & 9.2 & 11.5 & 10.5 & 9.9 & 11.1 & 10.4 \\
\hline & Controvertido & 9.2 & 4.5 & 4.4 & 4.8 & 5.6 & 5.6 \\
\hline & Medio & 59.8 & 57.8 & 57.5 & 57.8 & 60 & 58.3 \\
\hline \multirow[t]{5}{*}{ CHICAS } & Preferido & 6.5 & 12 & 12.4 & 12 & 1.5 & 10.3 \\
\hline & Rechazado & 2.5 & 7.6 & 4.6 & 7.7 & 3 & 5.8 \\
\hline & Ignorado & 14.1 & 15.8 & 13.7 & 15.9 & 10.4 & 14.8 \\
\hline & Controvertido & .5 & 2.1 & 2.6 & 3 & 9 & 2.5 \\
\hline & Medio & 76.4 & 62.5 & 66.7 & 61.4 & 76.1 & 66.6 \\
\hline \multirow[t]{5}{*}{ TOTAL } & Preferido & 6.8 & 10.6 & 12 & 11.7 & 5.1 & 9.9 \\
\hline & Rechazado & 9.1 & 12.4 & 10.8 & 12.3 & 10.2 & 11.3 \\
\hline & Ignorado & 11.4 & 13.6 & 12 & 12.9 & 10.8 & 12.4 \\
\hline & Controvertido & 5.3 & 3.4 & 3.6 & 4 & 7 & 4.2 \\
\hline & Medio & 67.4 & 60 & 61.7 & 59.2 & 66.9 & 62.1 \\
\hline
\end{tabular}

Los datos indican que los chicos obtienen porcentajes más altos de rechazo en todos los ciclos educativos, resultado que confirman otros estudios en donde se atribuye un mayor rechazo hacia los chicos. Por su parte, en el caso de las chicas, y aunque se observa mayores oscilaciones a lo largo de la escolaridad en su distribución sociométrica respecto de la que presentan los chicos, dominan los tipos ignorados y medios.

\section{¿La distribución sociométrica varía a lo largo de la escolaridad?}

Para estudiar la relación global entre ciclo y tipo sociométricos hemos empleado la prueba de $\chi^{2}$. Se han utilizado los residuos tipificados corregidos para encontrar las casillas en las que la relación entre las variables es difícilmente explicable a causa del azar. Se han tomado como referencia los valores \pm 1.96 . Como se observa en la tabla II, la relación global entre ciclo y tipo sociométricos es casi significativa, $\chi^{2}(16)=25.687 p=.059$. Es decir, que a lo largo de la escolaridad la proporción de cada tipo va cambiando, pero no de forma importante o no en todos los tipos. Los únicos cambios significativos son que el porcentaje de preferidos es menor en infantil y en secundaria y que el nivel sociométrico más indiferenciado se da en infantil. 
TABLA II

Tipo sociométrico y ciclo educativo

\begin{tabular}{|c|c|c|c|c|c|c|}
\hline \multirow[b]{2}{*}{ CICLO } & & \multicolumn{5}{|c|}{ TIPO SOCIOMÉTRICO } \\
\hline & & Preferido & Rechazado & Ignorado & Controvertido & Medio \\
\hline \multirow{3}{*}{$2^{\circ}$ Infantil } & $N$ & 30 & 40 & 50 & 23 & 295 \\
\hline & $\%$ Ciclo & 6.8 & 9.1 & 11.4 & 5.3 & 67.4 \\
\hline & Residuos & -2.4 & -1.6 & -.8 & 1.2 & 2.6 \\
\hline \multirow{3}{*}{ Inicial } & $N$ & 76 & 89 & 97 & 24 & 429 \\
\hline & $\%$ Ciclo & 10.6 & 12.4 & 13.6 & 3.4 & 60 \\
\hline & Residuos & .8 & 1.2 & 1 & -1.4 & -1.4 \\
\hline \multirow{3}{*}{ Medio } & $N$ & 40 & 36 & 40 & 12 & 206 \\
\hline & $\%$ Ciclo & 12 & 10.8 & 12 & 3.6 & 61.7 \\
\hline & Residuos & 1.4 & -.3 & -.3 & -.6 & -.1 \\
\hline \multirow{3}{*}{ Superior } & $N$ & 62 & 65 & 68 & 21 & 313 \\
\hline & $\%$ Ciclo & 11.7 & 12.3 & 12.9 & 4 & 59.2 \\
\hline & Residuos & 1.6 & .8 & .3 & -.3 & -1.6 \\
\hline \multirow{3}{*}{$1^{\circ} \mathrm{ESO}$} & $N$ & 8 & 16 & 17 & 11 & 105 \\
\hline & $\%$ Ciclo & 5.1 & 10.2 & 10.8 & 7 & 66.9 \\
\hline & Residuos & -2.1 & -.5 & -.7 & 1.8 & 1.3 \\
\hline
\end{tabular}

$\chi^{2}(16)=25.687 p=.059$

Los datos confirman que en la etapa infantil, los iguales no tienen tanta importancia y por tanto tampoco se da la apreciación de aceptación y rechazo de una manera tan acentuada como ocurre en ciclos educativos posteriores. Dentro de la etapa primaria, se inicia la comparación social entre iguales y con ello la referencia de si es mejor o peor compañero/a, que adquiere mayor importancia, si cabe, con el inicio de la etapa de educación secundaria.

¿La distribución sociométrica es igual para los chicos que para las chicas?

En la tabla III se presenta el porcentaje de cada tipo sociométrico en cada uno de los géneros. La asociación entre tipo sociométrico y género es significativa, $\chi^{2}$ $(4)=75.255 p=.000$. Los chicos son más rechazados y controvertidos que las chicas (16\% y $5.6 \%$ de los chicos y $5.8 \%$ y $2.5 \%$ de las chicas). Por su parte, las chicas son más ignoradas y mantienen más relaciones sociales promedio que los chicos (14.8\% y $66.6 \%$ de las chicas y $10.4 \%$ y $58.3 \%$ de los chicos).

\section{Interacciones entre los tres factores}

Las variaciones observadas de forma rápida en la tabla I, la diferente distribución sociométrica de chicos y chicas (Tabla III) y la variación casi significativa en el porcentaje de alguno de los tipos (Tabla II) hace que nos planteemos la hipótesis de si podría existir una interacción entre los tres factores. Para ello hemos realizado la comparación entre dos modelos loglineales. El primero incluía los tres pares de asociaciones posibles entre los tres factores, Ciclo x Tipo Sociométrico, Ciclo x Género y Género x Tipo sociométrico. El segundo incorporó además el efecto de la interacción entre las tres variables Ciclo x Género x Tipo sociométrico. La diferencia de ajuste de ambos modelos resultó estadísticamente significativa, $G^{2}(16)=27.054, p=.0409$. De esta forma confirmamos que las variacio- 
Distribución sociométrica en las aulas de chicos y chicas a lo largo de la escolaridad / F.-J. García-Bacete et al.

TABLA III

Tipo sociométrico y género

\begin{tabular}{llccccc}
\hline & & \multicolumn{5}{c}{ TIPO SOCIOMÉTRICO } \\
\cline { 3 - 6 } GÉNERO & & Preferido & Rechazado & Ignorado & Controvertido & Medio \\
\hline \multirow{2}{*}{ CHICO } & $N$ & 114 & 188 & 123 & 66 & 687 \\
& $\%$ & 9.7 & 16 & 10.4 & 5.6 & 58.3 \\
& Residuos & -.5 & 7.4 & -3.1 & 3.6 & -3.9 \\
\hline \multirow{2}{*}{ CHICA } & $N$ & 102 & 58 & 147 & 25 & 661 \\
& $\%$ & 10.3 & 5.8 & 14.8 & 2.5 & 66.6 \\
& Residuos & .5 & -7.4 & 3.1 & -3.6 & 3.9 \\
\hline
\end{tabular}

$\chi^{2}(4)=75.255 p<.000$

nes en los porcentajes sociométricos están afectadas por el género del alumno y por el ciclo educativo que cursa. Una relación de los efectos simples de asociación entre los tres pares de variables puede consultarse en la tabla IV. Dado que los porcentajes que van apareciendo en las tablas de contingencia son los mismos que los presentados en la tabla I, dispuestos de forma diferente en cada tabla, hemos decidido no presentarlas, y limitar los comentarios a los efectos significativos. Para estimar el tamaño de los efectos se ha hecho servir la razón de proporciones (Odds Ratio) (ver Tabla V)

TABLA IV

Efectos simples de asociación entre los tres pares de factores (ciclo, género, tipo sociométrico)

\begin{tabular}{lccc}
\hline Efecto & $g l$ & $\chi^{2}$ & n.s. \\
\hline Ciclo x Tipo sociométrico & 16 & 12.407 & .715 \\
Chico & 16 & 40.376 & .001 \\
Chica & & & \\
Ciclo x Género & 4 & 5.54 & .226 \\
Preferido & 4 & 6.335 & .175 \\
Rechazado & 4 & 1.445 & .836 \\
Ignorado & 4 & 10.823 & .029 \\
Controvertido & 4 & 2.229 & .694 \\
Promedio & & & \\
& 4 & 39.881 & .000 \\
Género x Tipo sociométrico & 4 & 19.810 & .001 \\
Segundo Infantil & 4 & 12.739 & .013 \\
Inicial Primaria & 4 & 12.255 & .016 \\
Medio Primaria & 4 & 11.074 & .026 \\
Superior Primaria & & & \\
Primero Secundaria & & & \\
\hline
\end{tabular}

$\mathrm{Al}$ analizar la asociación ciclo $\mathrm{x}$ tipo sociométrico para cada uno de los géneros, se observa que la distribución de los chicos en los diferentes tipos sociométricos es similar a lo largo de la escolaridad, mientras que en el caso de las chicas esta distribución varía, $\chi^{2}(16)=40.376 p=.001$. El porcentaje de chicas preferidas es más alto en primaria que en secundaria (12\% vs. $1.5 \%)$. La proporción media de niñas preferidas en primaria es unas 10 veces superior que en secundaria. En sentido contrario, hay menos chicas controvertidas en infantil y primer ciclo de primaria $(.5 \%$ y $2.1 \%)$ que en secundaria $(9 \%)$. El tamaño del efecto 
TABLA V

Componentes significativos de las interacciones de dos factores

\begin{tabular}{|c|c|c|c|c|c|c|}
\hline \multicolumn{4}{|c|}{ Niveles de los Factores que definen el efecto } & \multicolumn{3}{|c|}{ Intervalo del 95\% } \\
\hline Efecto & Género & Ciclo & Tipo & $\begin{array}{l}\text { Razón de } \\
\text { Proporciones }\end{array}$ & L Inferior & $\begin{array}{l}\text { Límite } \\
\text { Superior }\end{array}$ \\
\hline \multirow[t]{5}{*}{ Ciclo x Tipo } & $\mathrm{M}$ & I y $S$ & Cy Me & .06 & .01 & .048 \\
\hline & $\mathrm{M}$ & $1 \mathrm{PyS}$ & Py Me & 9.82 & 1.32 & 73.06 \\
\hline & $\mathrm{M}$ & $1 \mathrm{PyS}$ & Cy Me & .28 & .09 & .87 \\
\hline & $\mathrm{M}$ & $2 \mathrm{PyS}$ & Py Me & 9.5 & 1.24 & 72.97 \\
\hline & M & $3 \mathrm{PyS}$ & $\mathrm{Py} \mathrm{Me}$ & 9.99 & 1.32 & 75.29 \\
\hline Género x Ciclo & H у M & IyS & $\mathrm{C}$ & 26.4 & 2.57 & 271.09 \\
\hline \multirow[t]{6}{*}{ Género x Tipo } & Н у М & I & $\mathrm{R}$ y Me & 7.44 & 2.84 & 19.52 \\
\hline & Н у M & I & C y Me & 23.38 & 3.11 & 175.75 \\
\hline & Hy & $1 \mathrm{P}$ & $\mathrm{R}$ y Me & 2.39 & 1.46 & 3.92 \\
\hline & Ну М & $2 \mathrm{P}$ & $\mathrm{R}$ y Me & 4.06 & 1.70 & 9.69 \\
\hline & Hy M & $3 \mathrm{P}$ & $\mathrm{R}$ y Me & 2.20 & 1.22 & 3.95 \\
\hline & Hу $\mathrm{M}$ & $\mathrm{S}$ & $\mathrm{R}$ y Me & 6.61 & 1.43 & 30.54 \\
\hline
\end{tabular}

estimado es relativamente pequeño (.06 y .28 en infantil y primero de primaria). El porcentaje de chicas promedio va oscilando, pero resulta claro que son mas frecuentes en infantil y secundaria con porcentajes superiores al $75 \%$ que en Primaria con porcentajes entre 60-65\%. El porcentaje de niñas rechazadas e ignoradas es similar en todos los ciclos.

$\mathrm{Al}$ analizar la asociación ciclo $\mathrm{x}$ género para cada uno de los tipos sociométricos, se observa que a lo largo de la escolaridad tan sólo cambia la proporción de controvertidos/controvertidas, $\chi^{2}(4)=10.823 p=.029$, de forma que si en infantil la casi totalidad de controvertidos son chicos $(95.7 \%$ vs. $4.3 \%)$ en secundaria la proporción se ha invertido ( $45.5 \%$ vs. $54.5 \%)$. La razón de proporciones de niños controvertidos respecto de las niñas es 26.4 veces mayor en infantil que en secundaria. Esto es debido en parte a que el ser controvertido tiene mayor frecuencia relativa en niños que en niñas en el primer ciclo, y al contrario en el último.

$\mathrm{Al}$ analizar la asociación género $\mathrm{x}$ tipo observamos que en todos los ciclos educativos la distribución sociométrica de chicos y chicas es diferente (valores de $\chi^{2}$ significativas en todos los ciclos). En el segundo ciclo de infantil hay significativamente más chicos rechazados $(14.6 \%)$ y controvertidos $(9.2 \%)$ que chicas $(2.5 \%$ y $.5 \%$, respectivamente), siendo la proporción de niños rechazados 7.44 veces mayor que la de las niñas y 23.38 veces mayor la de niños-controvertidos que la de niñas-controvertidas. En el caso del rechazo la tendencia se mantiene en todos los ciclos, la proporción de chicos rechazados siempre es superior a la de las chicas, con unas razones de proporción estimadas de 2.39, 4.06, 2.20 y 6.61 sucesivamente desde el primer ciclo de primara al primero de secundaria.

Por último, cuando tomamos como "sujeto" cada una de las aulas y como "datos" los porcentajes de cada tipo sociométrico en esa aula, los resultados coinciden completamente con los descritos anteriormente, por lo que no vamos a presentarlos. Por el contrario, sí interesa destacar la gran variación en los porcentajes de cada tipo de unas aulas a otras (ver Tabla VI). De esta forma, se constata que la distribución concreta de cada tipo dependerá de las características concretas de cada grupo-aula y de su dinámica interna (Moreno, 1999). No obstante 
como se observa en la tabla, algunos de los hallazgos descritos siguen siendo evidentes: a) Sólo en el 8.7\% de las aulas el porcentaje de los chicos-rechazados es inferior al 5\%, mientras que en el caso de las chicas asciende a un 53.3\%; por la parte superior, en el $34.8 \%$ de las aulas el porcentaje de chicos-rechazados es superior al $20 \%$, mientras que en las chicas sólo ocurre en el $7.6 \%$ de las aulas. b) En la mitad de las aulas el porcentaje de chicos-controvertidos es inferior al $5 \%$, en el caso de las chicas esta situación se da en el $78.3 \%$ de las aulas. c) En el caso de los sujetos ignorados no hay diferencias en los intervalos bajos, sí en los altos; en el $43.4 \%$ de las aulas el porcentaje de chicas-ignoradas es superior al 15\%, en el caso de los chicos sólo ocurre en el $27.2 \%$ de las aulas.

TABLA VI

Distribución sociométrica por AULAS

\begin{tabular}{llcccccc}
\hline \multicolumn{7}{c}{ NÚMERO DE AULAS Y PORCENTAJES EN CADA INTERVALO } \\
\hline & $0-4.99 \%$ & $5-9.99 \%$ & $10-14.99 \%$ & $15-19.99 \%$ & $20-24.99 \%$ & $\begin{array}{c}25 \% \text { o } \\
\text { Superior }\end{array}$ \\
\hline CHICOS & Preferidos & $29(31.9)$ & $22(24.1)$ & $15(16.5)$ & $10(11)$ & $12(13.2)$ & $3(3.3)$ \\
& Rechazados & $8(8.7)$ & $16(17.4)$ & $19(20.6)$ & $17(18.5)$ & $14(15.2)$ & $18(19.6)$ \\
& Ignorados & $23(25)$ & $26(28.3)$ & $18(19.5)$ & $11(12)$ & $6(6.5)$ & $8(8.7)$ \\
& Controvertidos & $45(48.9)$ & $25(27.2)$ & $12(13)$ & $7(7.6)$ & $3(3.3)$ & 0 \\
\hline CHICAS & Preferidos & $28(30.4)$ & $19(20.7)$ & $21(22.8)$ & $8(8.7)$ & $9(9.8)$ & $7(7.6)$ \\
& Rechazados & $49(53.3)$ & $21(22.8)$ & $10(10.9)$ & $5(5.4)$ & $4(4.3)$ & $3(3.3)$ \\
& Ignorados & $21(22.8)$ & $13(14.2)$ & $19(20.6)$ & $9(9.8)$ & $10(10.9)$ & $20(21.7)$ \\
& Controvertidos & $72(78.3)$ & $10(10.8)$ & $7(7.6)$ & $3(3.3)$ & 0 & 0 \\
\hline TOTAL & Preferidos & $19(20.7)$ & $28(30.4)$ & $30(32.6)$ & $12(13)$ & $2(2.2)$ & $1(1.1)$ \\
& Rechazados & $10(10.9)$ & $27(29.3)$ & $33(35.9)$ & $19(20.6)$ & $3(3.3)$ & 0 \\
& Ignorados & $14(15.2)$ & $17(17.4)$ & $29(31.5)$ & $19(20.7)$ & $12(13)$ & $2(2.2)$ \\
& Controvertidos & $58(63)$ & $25(27.2)$ & $8(8.7)$ & $1(1.1)$ & 0 & 0 \\
\hline
\end{tabular}

\section{Discusión}

Los resultados encontrados nos permitirán tener una visión global de la presencia y evolución de los diferentes tipos sociométricos a lo largo de la etapa infantil y el inicio de la preadolescencia. Se han confirmado todas las hipótesis expresadas, a excepción de la distribución homogénea de los tipos sociométricos en todas las aulas. Iniciamos nuestra discusión por esta última cuestión.

En todas las aulas se producen relaciones entre los iguales y en todas las aulas encontramos alumnos de la mayoría de los tipos. Las relaciones entre iguales tienen efectos únicos en el desarrollo personal, social y escolar de los alumnos (Chan y Mpofu, 2002). Los motivos que chicos y chicas dan para afiliarse o no gustarse, la percepción de la conducta, el grado de conformismo con las normas del grupo de iguales o de la institución, el rendimiento académico (Wentzel y Asher, 1995) son algunos de los procesos que pueden explicar la situación sociométrica de cada chico y chica. También puede ser influida por los procesos de reputación, en los que el profesor desempeña un papel fundamental. Además, también es cierto que en cada aula se da una distribución sociométrica diferente, lo que nos hace pensar que las características y dinámica de cada aula son otro de los factores, y que la vida en las aulas no es completamente conocida por los profesores, pero no por ello deja de ejercer su impacto en donde los tipos sociométricos son tanto un resultado como un proceso interviniente. En este sentido, es importante que el profesor preste atención a las relaciones sociales, que conozca procedi- 
mientos de evaluación social como el sociotest de nominaciones que le permitan averiguar con qué tipo sociométrico se puede identificar a cada alumno y alumna, pero también que se pregunte porqué se da una distribución y no otra. García-Bacete et al. (1990) encontraron que los alumnos rechazados disfrutan menos de las actividades de clase, muestran insatisfacción con las ayudas que reciben y con la aplicación de las normas de clase.

Los porcentajes globales para cada tipo son: $9.9 \%$ preferidos, $11.3 \%$ rechazados, $12.4 \%$ ignorados, $4.2 \%$ controvertidos y $62.1 \%$ promedio. Chicos y chicas son sociométricamente diferentes en todos los ciclos. Ellos son más rechazados y controvertidos y ellas más ignoradas y promedio (Cillesen et al., 1996). El rechazo está claramente asociado al género masculino (García-Bacete, 2006). En todos los ciclos el porcentaje de chicos rechazados (en torno al 16\%) es claramente superior al de chicas, siendo la proporción de chicos hasta 7.44 veces superior a la de las chicas. La proporción de chicos-controvertidos también es superior a la de las chicas, pero está afectado por el ciclo. Por su parte, las chicas, no generan ni grandes simpatías ni grandes antipatías, especialmente en infantil y secundaria. De hecho, el porcentaje conjunto de chicas ignoradas y promedio oscila entre un $77.3 \%$ y un $90.5 \%$.

Por lo que se refiere al efecto ciclo, sólo la proporción entre chicos-controvertidos y chicas-controvertidas varía en la escolaridad. De hecho, desde porcentajes claramente superiores de los chicos en infantil se van igualando progresivamente hasta que en secundaria la proporción de chicas es superior que la de chicos. La distribución sociométrica de los chicos es estable durante toda la escolaridad, la de las chica cambia: 10 veces más preferidas en primaria que en secundaria y un poco más controvertidas en secundaria.

Formar parte de alguno de los grupos considerados no-preferidos tiene efectos negativos presente y futuros, lo que es especialmente cierto en el caso de los rechazados, en particular los varones-agresivos. Ahora bien, la escasa formación del profesorado en estos procesos y el que tanto los controvertidos como los ignorados suelen tener resultados académicos positivos o cuando menos promedio (Hatzichriston y Hopf, 1996), hace que los profesores no perciban la conducta negativa y las dificultades que los controvertidos experimentan en otros ámbitos, o que subestimen la timidez, aislamiento o tendencia a encerrarse en sí mismos de los ignorados.

Los controvertidos, aunque escasos en número, son un grupo de especial interés (García-Bacete, 2007). Este grupo suele ser tan agresivo como los rechazados $y$, en general, no suelen poseer mejores habilidades sociales que los rechazados ni características de liderazgo. Puede ser que su competencia académica actúe de forma compensadora, facilitando las relaciones con los profesores y la afiliación con los iguales. Por otra parte, no tener una visión completa y precisa de su comportamiento en todos los ámbitos, dificulta la posibilidad de integración. Algo parecido pasa con los ignorados, quienes son los preferidos de los profesores por su perfil académico alto, pero no son vistos como "buenos estudiantes" por sus compañeros. Puede ser que presenten mayores niveles de conformidad o de interés con las normas académicas que por las relaciones sociales, lo que justificaría tanto la aceptación por parte del profesor como el olvido por parte de los compañeros. Especialmente intensificado en el caso de las chicas, quienes son identificadas de manera mayoritaria en este tipo.

Esta situación a la que no se le ha considerado de riesgo durante los años de primaria, gana relevancia en la adolescencia, en donde la conformidad con las normas de grupo se hace más intensa, especialmente en el caso de los tipos más necesitados de apoyo social, como los rechazados y los ignorados y de modo especial en las chicas, en quienes se suman las dos condiciones (Oliva, 2002). Tam- 
bién en este periodo se modifica la asociación entre tipos y estereotipos de género (Hatzichriston y Hopf, 1996). Ello podría justificar el incremento de controvertidas y la disminución de ignoradas y preferidas en los primeros años de secundaria. Así, un porcentaje de chicas parece revelarse contra esa imagen positiva, pero también contra su falta de impacto, su perfil social medio o el ninguneo del que son objeto, sin que todavía estos comportamientos obtengan la aceptación por parte de sus compañeros ni del profesorado.

De cuanto venimos comentando, a modo de conclusiones, se pueden extraer una serie de posibles aplicaciones o recomendaciones que confirman la relevancia e interés del estudio realizado: 1) se dispone de un retrato de las posiciones sociométricas que ocupan los alumnos y alumnas a lo largo de la escolaridad; 2) son bien conocidos las consecuencias negativas que conlleva la falta de aceptación por parte de los iguales, y en especial de aquellos que padecen el ostracismo y la indiferencia por parte de su compañeros (Bierman, 2004; Wentzel y Asher, 1995) Los datos confirman que cerca de un $24 \%$ del alumnado (11.3\% de rechazados y $12.4 \%$ de ignorados) sufrirán estas consecuencias negativas. Se hace necesaria conocer la tipología sociométrica de los alumnos y alumnas y hacer una detección precoz de quienes se encuentran en situación de riesgo, especialmente los rechazados e ignorados, de forma que se puedan desarrollar estrategias psicoeducativas de intervención temprana, como son los programas de habilidades sociales y la incorporación de los contenidos socioafectivos en los curriculum escolares. 3) El estudio sociométrico dentro del aula permitirá conocer también las competencias interpersonales del alumnado. En este caso, los docentes podrán desarrollar un trabajo educativo y preventivo tanto frente a las conductas de violencia interpersonal (escolar, de género,...) como de las conductas pasivas o inmaduras. Se hace necesaria una enseñanza que atienda a las estrategias socioafectivas utilizadas dentro del grupo clase. 4) Conviene llamar la atención de los docentes y de los equipos psicopedagógicos sobre de la conveniencia de adoptar una visión más global de la realidad de las aulas, del contexto social, de las relaciones entre iguales. En particular, se trata de recuperar el papel del profesorado como líder del aula, consciente de que es quien estructura los contextos y actividades de aprendizaje y de su repercusión en las conductas, valores y actitudes que los alumnos adquieren. El profesorado tiene una influencia importante en los procesos de reputación social del aula y en las dinámicas sociales que operan en las aulas (Gifford-Smidt y Brownell, 2003). El que en cada aula se puedan observar distribuciones sociométricas diferentes avala el carácter interactivo de los tipos sociométricos, la importancia de cómo el contexto responde a las característica de los individuos (Bierman, 2004). 5) La distribución sociométrica identificada revela que en las aulas siguen vigentes los estereotipos sociales asociados al género, que los agentes de socialización, incluidos los propios niños y niñas, los profesores y las profesoras, a pesar de los avances, continúan ofreciendo un trato diferente a chicos y chicas de acuerdo con los mandatos de género (Díaz-Aguado, 2002; Sureda, García-Bacete y Monjas, en evaluación). Nuestros datos revelan que las chicas aparecen mayoritariamente como ignoradas y medias y los chicos como rechazados y controvertidos. La literatura revela con claridad la asociación entre masculinidad, rechazo y agresividad y la asociación entre feminidad, tipos ignorados y medios, inhibición, conformismo, sumisión y pasividad (Cillensen $e t$ al., 1996; Monjas, García-Larráuri, Elices, Francia y de Benito, 2005). El fuerte incremento de alumnas controvertidas al inicio de la secundaria y la disminución del número de ignoradas y promedio puede indicar que las preadolescentes empiezan a rebelarse contra el estereotipo asignado y empiezan a presentar conductas que no se corresponden con el estilo femenino y algunos y algunas no lo aceptan. En definitiva, es necesario formar a los docentes y equipos psicopedagó- 
gicos al menos en técnicas de identificación de los tipos sociométricos, en el diseño de contextos educativos social y emocionalmente saludables para los niños y las niñas y en la incorporación de programas de intervención que mejoren la convivencia en el aula.

\section{Referencias}

Arruga, A. (1983). Introducción al test sociométrico. Barcelona: Herder.

Bierman, K. (2004). Peer rejection. Developmental, processes and intervention strategies. New Cork: The Guilford Press.

Cava, M. J. \& Musitu, G. (2001). Autoestima y percepción del clima escolar en niños con problemas de integración social en el aula. Revista de Psicología General y Aplicada, 54 (2), 297-311.

CHAN, S. \& MPOFU, E. (2002) Children's peer status in school settings. Current and prospective assessment procedures. School Psychology International, 22 (1), 43-52

Cillesen, A. H. N. \& Bukowsli, W. M. (2000). Conceptualizing and measuring peer acceptance and rejection. En A. H. N. Cillessen \& W. M. Bukowski (Eds.), Recent advances in the measurement of acceptance and rejection in the peer system (pp. 3-10). San Francisco, CA: Jossey-Bass Inc.

Cillesen, A. H. N., Coie, J., Terry, R. \& Lochman, J. E. (1996). The role of gender in the behavior basis of children's sociometric status evaluations. Paper presented at the biennial meetings of the International Society for the Study of Behavioral Development, Quebec City, Quebec, Canada.

Díaz-Aguado, M. J. (2002). Prevenir la violencia contra las mujeres: construyendo la igualdad. Programa para educación secundaria. Madrid: Instituto de la Mujer.

DunCAN, M. K. \& COHEN, R. (1995). Liking within the peer group as a function of children's sociometric status and sex. Child Study Journal, 25 (4), 265-287.

GARCÍA-BACETE, F. J. (2006). La identificación de los alumnos rechazados. Comparación de métodos sociométricos de nominaciones bidimensionales. Infancia y Aprendizaje, 29 (4), 437-451.

GARCÍA-BACETE, F. J. (2007). La identificación de los alumnos rechazados, preferidos, ignorados y controvertidos en el aula. Revista de Psicología General y Aplicada (aceptado, 27-12-06).

García-BACEte, F. J., GARCiA, D. \& SuredA, I. (2005). Los motivos de aceptación entre iguales: Un intento de categorización y de establecer diferencias entre género. En I. Fajardo, F. Vicente, A. Ventura, I. Ruíz \& J. A. del Barrio (Eds.), Aportaciones psicológicas y mundo actual dando respuestas (pp. 269-279). Badajoz: Psicoex.

García-Bacete, F. J., Lara, A. \& Monjas, I. (2005). ¿Por qué los niños no quieren jugar con otros niños? Un análisis exploratorio de los motivos de rechazo entre iguales. En I. Fajardo, F. Vicente, A. Ventura, I. Ruíz \& J. A. del Barrio (Eds.), Aportaciones psicológicas y mundo actual dando respuestas (pp. 257-268). Badajoz: Psicoex.

García-Bacete, F. J., Musitu, G. \& García, M. (1990). El perfil ecológico del alumno rechazado en EGB. Actas del III Congreso de Psicología Social (pp. 324-344). Santiago de Compostela: Universidad de Santiago.

GifFord-Smith, M. E. \& Brownell, C. A. (2003). Childhood peer relationships: Social acceptance, friendships and peer networks. Journal of School Psychology, 41, 235-284.

GÓNZALEZ, J. (1990). Sociometría per ordinador. El test sociometric. Valencia: Conselleria de Cultura, Educació i Ciència.

GónZAlez, P. (1998). Las medidas sociométricas como indicadores de la competencia social en la infancia. Psicologemas, 12 (23), 93-122.

HATZICHRISTON, CH. \& Hopf, D. (1996). A multiperspective comparison of peer sociometric status groups in childhood and Adolescence. Child Development, 67, 1085-1102.

Jimenez, M. (2003). Características emocionales y comportamentales de los grupos sociométricos desde una perspectiva múltiple. Psicología Conductual, 11 (1), 41-60.

LEGAULT, F. (1993). Gender differences in the perceptions of affiliative networks in primary school. Poster presented at the biennial meeting of the Society for Research in Child Development, New Orleans, LA.

Monjas, M. I., García-Larráuri, B., Elices, J. A., Francia, M. V. \& DE Benito, P. (2005). Aceptación social y género en la infancia y en la adolescencia. En I. Fajardo, F. Vicente, A. Ventura, I. Ruiz \& J. A. del Barrio, Aportaciones psicológicas y mundo actual. Dando respuestas (pp. 383-393). Badajoz: Psicoex

Moreno, M. C. (1999). Desarrollo y conducta social de los 6 años a la adolescencia. En J. Palacios, A. Marches \& C. Coll (Comps.), Desarrollo psicológico y educación. Tomo I, Psicología Evolutiva (pp.405-430). Madrid: Alianza Editorial

Newcomb, A. F., Bukowski, W. M. \& PAtTEe, L. (1993). Children's peer relations: a meta-analytic review of popular, rejected, neglected, controversial and average sociometric status. Psychological Bulletin, 113, 99-128.

Oliva, A. (1999). Desarrollo social durante la adolescencia. En J. Palacios, A. Marches \& C. Coll (Comps.), Desarrollo psicológico y educación. Tomo I, Psicología Evolutiva (pp. 493-517). Madrid: Alianza Editorial

Ortíz, M.J., Aguirrezabala, E., Apodaka, P., Etxebarria, I. \& López, F. (2002). Características emocionales, funcionamiento social y satisfacción social en escolares. Infancia y Aprendizaje, 25 (2), 195-208.

RoDrígueZ, A. \& MORERA, D. (2001). El sociograma. Estudio de las relaciones informales en las organizaciones. Madrid: Pirámide.

SuREDA, I., GARCíA-BACETE, F. J. \& MONJAS, I. (2007 en evaluación). Razones que dan chicos y chicas para preferir y rechazar a sus iguales. Revista Latinoamericana de Psicología

Trianes, M. V., Blanca, M. J., Muñoz, A., García, B., Cardelle-Eleawar, M. \& Infante, L. (2002). Relaciones entre evaluadores de la competencia social en preadolescentes: profesores, iguales y autoinforme. Anales de Psicología, 18 (2), 197214.

Villanueva, L., Clemente, R. A. \& García-Bacete, F. J. (2002). La comprensión infantil de la mente y su relación con el problema del rechazo entre iguales. Infancia y Aprendizaje, 25 (1), 85-100.

WENTZEL, K. R. \& AsHeR, S. R. (1995). The academic lives of neglected, rejected, popular, and controversial children. Child Development, 66, 754-763. 